\title{
AN EFFICIENT MONITORING SYSTEM FOR SPORTS PERSON USING WI-FI COMMUNICATION
}

\author{
P.Santhosh Kumar ${ }^{1}$, V.Arun Kumar ${ }^{2}$ \\ ${ }^{1}$ ME-Embedded System Technology, Nandha Engineering College, Erode, Tamilnadu, India \\ ${ }^{2}$ Asst Professor - EEE department, Nandha Engineering College, Erode, Tamilnadu, India
}

\begin{abstract}
The data from athletes body-sensor devices that monitor personal metrics like heart-rate, weight, and movements are changing how athletes train and perform. Existing sport-analytic tools are, however, mostly monolithic proprietary systems where athletes have little control over how their data is used and managed over time. To monitor the sportsperson metrics and Blood pressure value in playground. Optimal solution by using Wi-Fi to monitor the human BP and movements changing human body with the help of the sensor The monitored values will be shown in a LCD display.
\end{abstract}

Keywords -WiFi, girji, human bp, pic controller

\section{INTRODUCTION}

The embedded devices monitor and record daily individual physiological metrics like heart rate, weight, and movements, are becoming ubiquitous. Large-scale analytic of such body sensor data from quantified athletes is reshaping how sports are played and how athletes are being developed. Online real time publication of detailed and precise game information and statistics also enable supporters to engage with their favorite sport at a completely new level. As body sensor technology advances, more athlete parameters become available for quantification and at an increasing level of precision.

Collecting, storing, analyzing, and correlating large volumes of personal body sensor data from a large cohort of athletes, enables coaches and medical staff to find normal and abnormal performance and health indicators that might not be visible from studying single records alone [2]. The ability to extract useful high-level signals from voluminous data, known as big-data analytic, is important for the emerging next generation sports analytic systems. By extending our sensing platform with available consumergrade self-tracking devices like the Fit-Bit Flex arm- band and the Run Keeper mobile-phone applications, we can acquire a more complete coverage of athlete telemetry. Because such sensing devices are made for the nontechnical consumers, they are easy to use and come with hard-wired data upload facilities and functional online user interfaces. This enables small sports clubs like TIL to make use of such devices for athlete quantification with little cost. Unfortunately, this also means that athletes are required to store potentially sensitive personal metrics like weight, blood pressure, position, and energy expenditure in proprietary data silos [6] outside their primary control sphere.

Although having personal data stored in numerous data silos is part of the digital age, concerns are already being raised that people are losing control of their data. In a study from 2010, $72 \%$ of Internet users in Europe say they think too much personal information is stored online [7]. The European Commission has already stated that individuals have the right to control their data, including the right to be forgotten [], and extending body-sensor data acquisition out beyond the sports arena might infringe upon athletes rights to control their own information. This is to a little extent addressed by existing sport-tracking systems used by professional sports clubs or consumer systems targeting the more casual users.

Emerging online services like the Microsoft Health Vault and Run Keeper already allow athletes and other users to store body-sensor data in online data silos. This trend of using online services to collect and host personal data is prevalent and something that is likely to stay. There is also many benefits associated with storing data in professionally managed services, including increased availability and regular backups. Professionally managed services are generally also better equipped and skilled to prevent data leakage due to hacker intrusions. Our conjecture is that, if enabled to do so in a safe and privacy preserving manner, the quantified athletes will consent to having parts of their body-sensor data archives made avail- able to relevant sport and health related data consumers like the sports club's coaching staff or large-scale medical cohort studies like the Troms $\emptyset$ study [9]. The consent will be given more willingly if the outcome of such participation is directly beneficial to the athlete, as in customized exercise programs, personalized diets; advancing sports and health research; or improving individual or team performance.

In this paper, describing Girji, a big-data analytic infrastructure that makes consent a first-order operational object that encapsulates and controls access to body-sensor data. Girji is designed to support a wide range of sensor and data types, and data analytic purposes. 


\section{BODY-SENSOR SYSTEMS}

The telemetry from body-sensor devices and other similar sources are generally not directly accessible to third-party Analytical Principals (APs). Instead, such systems are often hard-wired to upload captured body-sensor data to back-end sensor specific storage silos, often using convenient wireless communication technologies like Bluetooth, nearfield communication, or Wi-Fi. The primary role of Girji is therefore to acts as an intermediate and partially trusted broker between the athletes that produce body-sensor data, and the numerous APs that want to make use of this data. Girji will only allow an AP to access $p$ 's data if it is acceptable according to $p$ 's operational consent, which essentially is a private, programmable, and extensible personal reference monitor, which we also refer to as his Consent Object (CO). Another important part of Girji is, as with most other big- data infrastructures, our data acquisition facilities.

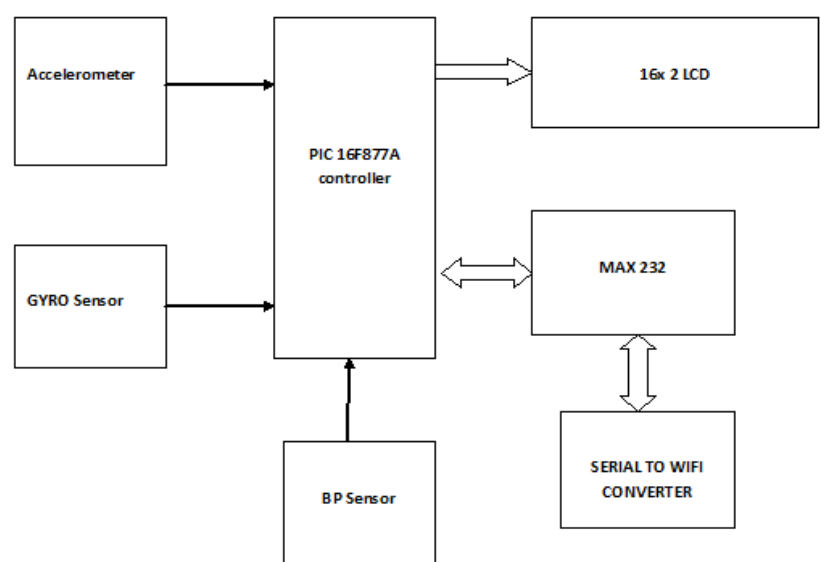

Fig 1 Monitoring system using WI-FI

We are particularly concerned with collecting data from athletes, often with non-technical backgrounds and with little time to tinker with gadgets and computers, significant effort is often needed to build reliable sensor products with well-designed user interfaces that can easily be adapted in the daily activities of the athletes and their sports clubs. Instead of building custom solutions for acquisition, a novel aspect of Girji is that we leverage the large number of existing sensors systems already available both in the professional sport market and in the consumer market. These systems take care of user interaction, including hardware and firmware maintenance, graphical interfaces, and end-user support.

\subsection{The ZXY System}

Each stationary radio receiver computes the position data for each player using vector-based processing of the received radio signals from the active belts. This enables direct projection of each player's position on the field without the use of conventional triangulation methods. The default positional sampling rate per belt is currently set to $20 \mathrm{~Hz}$, transmitting to a central relational database for storage in close to real- time. The producer of ZXY claims accuracy in the order of 1 meter for our version of the system, which conform to our own experiences and is significantly more accurate than more traditional GPS based tracking solutions. In addition to positional data, the belt includes an accelerometer that registers body movements in all three directional axes, a small gyroscope, a heart-rate sensor, and a compass. The accelerometer provides valuable data in addition to the more common data of distance covered in different speed categories.

Data from the sensor belt is aggregated and stored in a central relational database. By including all body sensor information in the same radio signal used for computing the positions, the system enables time synchronization of all raw data when stored in the database.

\subsection{RunKeeper}

The athlete tracking systems like ZXY provide valuable and accurate data for analytic in Girji, its usage is confined to the stadium where it is installed. To achieve more complete coverage of the athletes telemetry, Girji has built in connectors for many consumer body-sensor systems, like the RunKeeper and the more recent Fit Bit Flex. Unlike the professional systems, like ZXY, these consumer systems are mostly hard-wired to upload data to specific we services. We consider this a benefit since it reduces the level of tinkering needed to operate the devices correctly. This does, however, imply that Girji cannot obtain all relevant data from one single central repository. Instead, we must access data scattered over multiple data silos ranging from personal computers and special purpose sensor systems, like ZXY, to consumer targeted web services like Fit Bit. It is not likely that athletes are willing to spend time manually uploading their data into Girji. Instead, we acquire this data using a special purpose body-sensor data crawler that can access individual data silos using their site-specific data extraction APIs, which many online data silos are now providing gives an overview of some of the web services that we are currently using.

An example of a consumer-level data source is RunKeeper. Using their open Health Graph API, Girji can access many types of body-sensor data, like fitness activities, weight and sleep measurements, stored within their service. Access to this data is managed using the OAuth 2.0 authentication protocol, which is commonly used by other Internet services like Facebook, Twitter, and Google to authenticate and authorize third-party applications. The benefit of using OAuth 2.0 is that users can give third-party applications like Girji access to their RunKeeper account without having to share their credentials with the applications.

To access data in RunKeeper, we therefore first need to register Girji as an application in the Health Graph system. After providing RunKeeper with the name of our application and the URL of our web-site, Girji will receive an identity token and a secret. When the athlete wants Girji to access his Health-Graph data, he simply adds our application to his RunKeeper account. 
Although consumer devices and services are cost-effective and convenient, they cannot replace professional system shalll together. There are two primary reasons for that. First, professional systems offer higher precision than consumer systems. Secondly, the upstream and downstream capacity of consumer systems are much lower than professional body sensor systems. For instance, the ZXY system captures positional data at a rate of $20 \mathrm{~Hz}$ in real-time for all 22 players in a soccer match. The overhead of data transfer in RunKeeper is significant when the number of data points increase, which makes simultaneous high-frequency data capture from many sensors difficult to achieve.

\section{OPERATIVE CONSENT}

Body-sensor data often contains information that users consider highly sensitive and personal, like heart-rate and sleep patterns. We have a principled approach to privacy by design and participation in analytic projects with personal body-sensor data should have a strong notion of voluntarily associated with it. We therefore have built into the core of Girji a strong notion of informed and active consent associated with the collected data. In essence, such a consent is an individual's autonomous authorization of an intervention or participation in some project where private information might be disclosed and used. In the field of medicine, it is already well established that physicians or researchers must obtain such consent from their subjects. Those that are to obtain the consent have an obligation to tell the subject about the procedure of the participation, the potential risks, and benefits to the subject.

The subject should not be deceived or coerced, which implies that the subject must adequately comprehended the consent form. Finally, the subject must intentionally sign the consent form. Traditional consents in the form of passive paper documents, stated and signed at the time data is first collected, does however not capture well the dynamic protection and consensual agreement needed for long term usage of body sensor data. For instance, the athlete might change his mind in what he wants to provide and for what purpose. This could be a result of emerging devices collecting new or more accurate data. With a large number of users and projects, managing user consent in such a dynamic setting quickly becomes cumbersome, consuming significant resources.

To automate consent management and provide long term protection of body-sensor data, Girji introduces the notion of operational consent. Essentially, this is an extensible per user sandbox or reference monitor in the form of a CO that manages data access on behalf of each athlete. An architectural overview of Girji can be found.

The project description may contain both structured and unstructured textual data that describes the terms in human readable text. This text is then indexed and cataloged by Girji, or in the case of more public projects, also by a websearch engine like Google. Users interested in providing data can browse or search the list of project descriptions to find a candidate AP they want to support. Once a project is found, athletes can download the CRO and check his eligibility and data archive, as described next

\subsection{Extensibility using Meta-Code}

Because Girji is intended to manage athletes' data over long periods of time, users must be able to adapt their personal $\mathrm{COs}$ as their individual requirements change. To support a high degree of user customization, the core of each $\mathrm{CO}$ consists of an extensible run-time environment based on the Sourgi meta-code execution engine. This enables users to submit personalized code snippets that attach to data segments and can, for instance, be configured to execute each time an access attempt is made. The meta-code can scan the data it is associated with and its structure to make sure the consent assigned to the data is not violated. This in the context of the moment just before it is used. Policies can be hard-coded directly in the meta-code, as illustrated with the example in Fig.4,or fetched at run-time, which enables dynamic revocation or modification of the consent. In the event that a rule is broken, the meta-code will propagate a data-access exception back to the user and also created_on = datetime. utcfromtimestamp(self.metadata['created_on'])

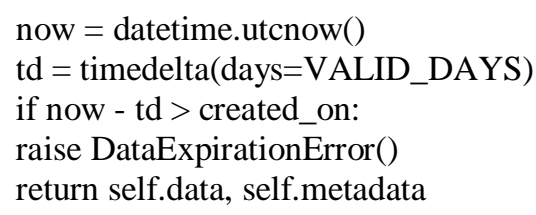

Alternatively to the requester with an explanation why the data cannot be accessed. It might also encode information about how to remedy the situation by, for instance, providing information on how the correct access tokens can be obtained, suggesting modifications to the extraction function so it no longer triggers a rule break, or requesting a modification to the policies within the $\mathrm{CO}$. The meta-code is packaged with the data that is released to the AP, and the Sourgi execution engine must be installed on their storage.

\subsection{Data Access and Delegation}

Access to athlete's data in Girji is managed through our data processing infrastructure, which use highly expressive bearer tokens to specify and manage secure analytic pipelines .Because object capabilities, as used in Girji, do not require centralized management and authentication, they are better suited for managing access control in decentralized data-sharing environments compared to the more traditional approaches based on Access Control Lists (ACLs), as for instance in the PDV system. Simple password schemes with e-mail verification, as used in MyPHR Machines, do not support confined delegation of access rights and are as such not well suited to enforce the principle of least privileges.

In Girji, each athlete $\mathrm{p} 1$ is given a root access token to all his data stored within his $\mathrm{CO}$, which includes the rights to read, write, and delete this data. An AP pn can only obtain restricted rights to access this data through chain, or a heritage, of connected data processing functions, where $\mathrm{f} 1$ is 
specified by $\mathrm{p} 1$. The heritage is signed using a Hash-based Message Authentication Code (HMAC) and taking a secret key into calculation so that it cannot be tampered with. The previous step in the chain and the governing operational consent of $\mathrm{p} 1$, embedded in his $\mathrm{CO}$ from where the data originates. Having the heritage hn, only principal pn can initiate data access using the API call. Receiving this call, Girji will first verify the integrity of hn using its signature, then start applying the contained functions to p1's data in the specified order of delegation.

\section{CONCLUSION}

Different types of body-sensor systems are currently available to sports clubs for tracking their athlete performance.

Existing systems range from professional stationary high precision tracking systems to consumer grade gadgets, like the Fit Bit Flex armband and mobile phones with applications like RunKeeper. The resulting constellation of devices and Internet services makes it difficult for sports clubs and other epidemiological data consumers to acquire and make use of body-sensor data from a cohort of users while at the same time ensuring that user consents are not violated. To prevent consent violations, the Girji system that we are currently developing in collaboration with TIL, a Norwegian soccer club, makes consent an operational firstclass object that encapsulates and controls the individual body-sensor data archives for each user.

\section{REFERENCES}

[1]. P. Dizikes, "Sports analytics: a real game-changer," Massachusetts Institute of Technology, MIT News Mar. 4, Mar. 2013.

[2]. I. Steadman, "IBM's Watson is better at diagnosing cancer than human doctors," Wired magasine, http://www.wired.co.uk, Tech. Rep., Feb.2013.

[3]. D. Johansen, M. Stenhaug, R. B. A. Hansen, A. Christensen, and P.-M. Høgmo, "Muithu: Smaller footprint, potentially larger imprint," in Proc. of the 7th International Conference on Digital Information Management. IEEE, Aug. 2012, pp. 205-214.

[4]. H. K. Stensland, V. R. Gaddam, M. Tennøe, E. Helgedagsrud, M. Næss,H. K. Alstad, A. Mortensen, R. Langseth, S. Ljødal, $\varnothing$. Landsverk,C. Griwodz, P. Halvorsen, M. Stenhaug, and D. Johansen., "Bagadus: An integrated real-time system for soccer analytics," ACM Transactions On Multimedia Computing, Communications and Applications, 2014.

[5]. H. D. Johansen, S. A. Pettersen, P. Halvorsen, and D. Johansen," Combining video and player telemetry for evidence-based decisions in soccer," in Proc. of the 2nd International Congress on Sport Sciences Research and Technology Support (icSport). SCITEPRESS Digital Library, Sep. 2013.

[6]. E. Singer, "The measured life," in MIT Technology Review, Jul. / Aug.2011.
[7]. European Commission, Special Euro barometer 359: Attitudes on Data Protection and Electronic Identity in the European Union, Dec. 2010.

[8]. "Safeguarding privacy in a connected world a European data protection framework for the 21 st century," in COM(2012)9. EURLex,Sep. 2012.

[9]. B. K. Jacobsen, A. E. Eggen, E. B. Mathiesen, T. Wilsgaard, andI. Njølstad, "Cohort profile: The Tromsø study," International Journal of Epidemiology, 2011.

[10]. M. Mohr, P. Krustrup, and J. Bangsbo, "Match performance of high standard soccer players with special reference to development of fatigue," Journal of sports sciences, vol. 21, no. 7, pp. 519-528, Jul.2003.

[11]. Fitness Keeper, Inc., "Health Graph API," website http://developer.runkeeper.com, Nov. 2013. 